\title{
Study on Electromagnetic Transient Condition of EMU Passing by Phase-separation with Electric Load in High-speed Railway*
}

\author{
Xiaoxu Guo, Jianzhong Wei, Long Xu, Shibin Gao, Zhengqing Han \\ School of Electrical Engineering, Southwest Jiaotong University, Chengdu, China \\ Email: guoxiaoxu198805@126.com
}

Received February, 2013

\begin{abstract}
Aiming at the complex electromagnetic transient process of EMU passing by phase-separation with electric load in high-speed railway, mechanism of overvoltage caused by switching off, overvoltage caused by switching on and impact current is analyzed systematically in this article. $\pi$-type equivalent circuit of feeding section is put forward in the analysis of overvoltage mechanism. Overvoltage and overcurrent model of passing by phase-separation with electric load are also built. Correctness of mechanism was validated by simulation. In addition, the methods to solve the influence on substations, transformers and protection devices in this process are put forward, which provides a new idea on passing by phase-separation with electric load technology.
\end{abstract}

Keywords: Passing by Phase-separation with Electric Load; Electromagnetic Transient Process; Overvoltage; Impact Current; Traction Power Supply System

\section{Introduction}

To make the balance of three phase load, the power supply of phase sequence rotation among substations is used in the traction power supply system. At the same time, in order to realize the electrical isolation of feeding section, the phase-separation is added in the output of the traction substation and section post in every 20-30 km [1]. In order to meet the requirements of high-speed operation and security, the way of ground-switch automatic convert passing by phase-separation with electric load has been adopted in high-speed railway [2]. Ground-switches are being switched frequently when EMU passing by phase-separation with electric load, and electrical parameters of "traction network - EMU - phase separation system" are changing ceaselessly in this process, which make traction network, EMU and phase separation system operate from one state to another continuously. At the same time, electromagnetic parameters in the system such as voltage and current are changing complicated in this dynamic process, which have an influence on various components of the traction power supply system [3-7]. There are some researches about complex electromagnetic transient process of EMU passing by phase-separation with electric load. That overvoltage caused by

\footnotetext{
*Supported by China National Natural Science Fund (50907055, U1134205 and 51177139) and Fundamental Research Funds for the Central Universities
}

switching off, overvoltage caused by switching on are analyzed in theory and using electronic switch to avoid overvoltage is put forward as in [8]. Transient response of the system when locomotive transformer is invested is analyzed as in [9], and selecting the appropriate voltage phase angle into the locomotive transformer is to reduce the inrush current. Reference [10] put forward that secondary harmonic content of differential current is reducing because of the differences of CT transmission characteristics in both sides of transformer when closed with load.

Based on this, mechanism of all kinds of the transient phenomena is researched systematically, and overvoltage and overcurrent model of passing by phase-separation with electric load are also be built. Electromagnetic transient process having an influence on traction supply system is studied through analysis of simulation.

\section{Scheme of Ground-switch Automatic Passing by Phase-separation}

As ground-switch automatic passing by phase-separation representative Shinkansen in Japan adopts the mode of insulated overlaps device, which not only improves the quality of pantograph's current collection, but also takes a short time, usually between $0.1 \sim 0.15 \mathrm{~s}$ [11]. In this way, the overhead catenary system(OCS) has no power supply dead zone, and the main circuit breaker on EMU does not 
need action, therefore passing by phase-separation with electric load is achieved. Its operational principle is shown in Figure 1.

The neutral section is set up at phase-separation where insulators $F_{1}, F_{2}$ are installed to insulate OCS in different phase. Vacuum circuit breakers $\mathrm{QF}_{1}, \mathrm{QF}_{2}$ are across-theline in two different phases separately. Four locomotive position sensors $\mathrm{CG}_{1} \sim \mathrm{CG}_{4}$ are installed on both sides of the rail. The neutral section is uncharged when $\mathrm{QF}_{1}, \mathrm{QF}_{2}$ are turned off when locomotive sails into neutral section, and sensor $\mathrm{CG}_{1}$ can sense the position of locomotive, neutral section is supplied by A phase in the event that $\mathrm{QF}_{1}$ is turned on. When the locomotive arrives at $\mathrm{CG}_{2}$ but not reaches $\mathrm{CG}_{3}, \mathrm{QF}_{1}$ is turned off and $\mathrm{QF}_{2}$ is turned on, so neutral section is supplied by $\mathrm{B}$ phase. When sensor $\mathrm{CG}_{4}$ can sense the position of locomotive, $\mathrm{QF}_{1}$ and $\mathrm{QF}_{2}$ are turned off, so neutral section returns to the state that the locomotive does not go through.

\section{Analysis of Electromagnetic Transient in EMU Passing by Phase-separation}

\subsection{Transient Process Equivalent Model}

Electrical model in transient process mainly includes three parts, such as OCS, neutral section and EMU. OCS and neutral section are distributed parameter circuits in essence, which is the same as electric power line. OCS can be expressed as $\pi$-type equivalent circuit, and neutral section can be expressed as T-type equivalent circuit. The main transformer on EMU is represented as RL series impedance. On the basis of the equivalent circuit of the three parts above-mentioned, equivalent power supply and equivalent impedance of the traction substation as well as mutual capacitance between OCS and neutral section are considered. Equivalent model of EMU passing by phase-separation is shown in Figure 2.

In the scheme, $\mathrm{U}_{\mathrm{A}}$ and $\mathrm{U}_{\mathrm{B}}$ represent equivalent power supplies of the traction substation. $\mathrm{R}_{\mathrm{S} 1}, \mathrm{R}_{\mathrm{S} 2}$ and $\mathrm{L}_{\mathrm{S} 1}, \mathrm{~L}_{\mathrm{S} 2}$ represent separately equivalent resistance and equivalent inductance of the two power supplies. $\mathrm{R}_{1}, \mathrm{R}_{2}$ and $\mathrm{L}_{1}, \mathrm{~L}_{2}$ represent separately equivalent resistance and equivalent inductance of the two feeding section. $\mathrm{C}_{1}, C_{1}^{\prime}$ and $\mathrm{C}_{2}$,

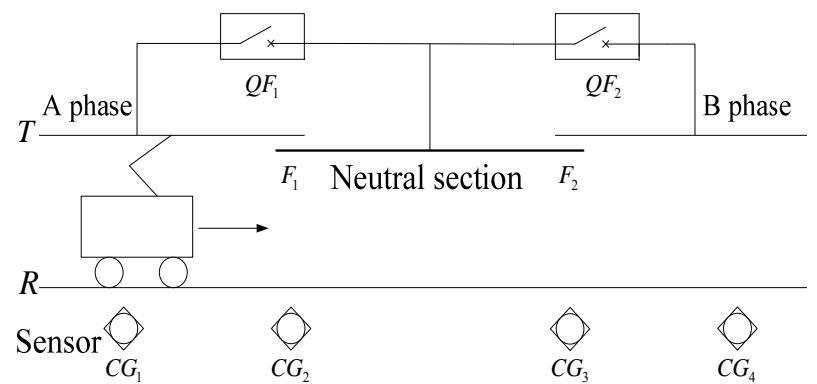

Figure 1. Schematic diagram of automatic passing phaseseparation with ground switch.
$C_{2}^{\prime}$ represent ground capacitance of the left and right feeding section separately. $\mathrm{R}_{\mathrm{N} 1}, \mathrm{R}_{\mathrm{N} 2}$ and $\mathrm{L}_{\mathrm{N} 1}, \mathrm{~L}_{\mathrm{N} 2}$ are separately equivalent resistance and equivalent inductance of the neutral section. $\mathrm{C}_{\mathrm{N}}$ represents ground capacitance of the neutral section. $\mathrm{C}_{\mathrm{N} 1}$ and $\mathrm{C}_{\mathrm{N} 2}$ represent mutual capacitance between OCS and neutral section. $\mathrm{QF}_{1}$ and $\mathrm{QF}_{2}$ represent vacuum circuit breakers. $\mathrm{R}_{3}$ and $\mathrm{L}_{3}$ represent separately equivalent resistance and equivalent inductance of the locomotive's transformer. $\mathrm{C}_{3}$ represents the ground capacitance of locomotive.

\subsection{Mechanism Analysis of Overvoltage Caused by Switching off}

Because the locomotive is inductive load, current is suddenly cut off before crossing zero when circuit breaker $\mathrm{QF}_{1}$ is turned off. The residual electromagnetic energy in the inductor is converted to capacitor energy because of inductor current charging the capacitor, which results in the sharp rising of the capacitor voltage. That is why the overvoltage caused by switching off appears. overvoltage generated interceptor. The influence of locomotive resistance $R_{3}$ is ignored. The simplified circuit schematic is shown in Figure 3.

Making $\mathrm{L}=\mathrm{L}_{3}, \mathrm{C}=\mathrm{C}_{\mathrm{N}}+\mathrm{C}_{3}, \mathrm{QF}_{1}$ is turned off when I $=\mathrm{I}_{\mathrm{m}} \sin \theta$, equation of oscillation circuit has been got as follow.

$$
C \frac{d u}{d t}+\frac{1}{L} \int u d t=0
$$

general solution as in

$$
u=a_{1} \cos \frac{1}{\sqrt{L C}} t+a_{2} \sin \frac{1}{\sqrt{L C}} t
$$

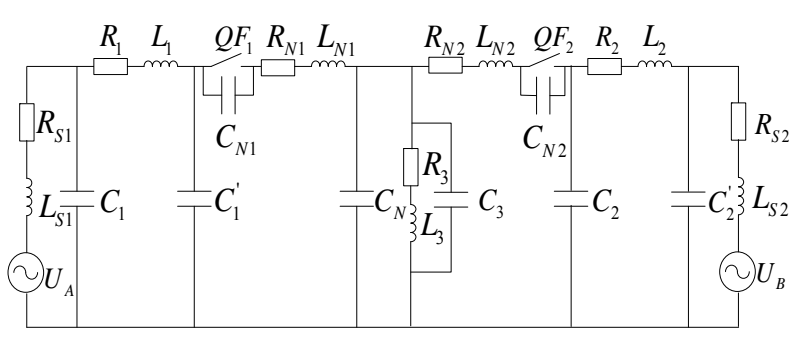

Figure 2. Equivalent modeling schematic diagram of EMU passing phase-separation.

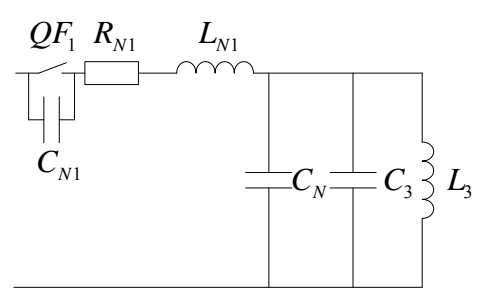

Figure 3. Schematic diagram of equivalent circuit when $\mathbf{Q F}_{1}$ is turned off. 
In ideal conditions, the magnetic energy and the electric energy are oscillating in the entire loop, and the angle frequency of the oscillation is $\omega_{0}=\frac{1}{\sqrt{L C}}$.

Initial condition is

$$
\left\{\begin{array}{l}
u(0)=-U_{0}=-U_{m} \cos \theta \\
i(0)=-\left.C \frac{d u}{d t}\right|_{t=0}=I_{0}=I_{m} \sin \theta
\end{array}\right.
$$

where, $-\mathrm{U}_{0}$ represents initial voltage of capacitor; $\mathrm{I}_{0}$ represents current of inductor in the moment $\mathrm{QF}_{1}$ is turned off.

Make (3) into (2)

$$
u=-U_{0} \cos \omega_{0} t-I_{0} \sqrt{\frac{L}{C}} \sin \omega_{0} t
$$

where, $-\mathrm{U}_{0} \cos \omega_{0}$ represents oscillation component caused by electric energy in $\mathrm{C} ;-I_{0} \sqrt{\frac{L}{C}} \sin \omega_{0} t$ represents oscillation component caused by magnetic energy in $\mathrm{L}$.

$$
U_{m}=\sqrt{I_{0}^{2} \frac{L}{C}+U_{0}^{2}}=I_{0} \sqrt{\frac{L}{C}+\frac{U_{0}^{2}}{I_{0}^{2}}}
$$

where, $\mathrm{U}_{0}$ is very small and $\frac{U_{0}^{2}}{I_{0}^{2}} \approx 0$, so $U_{m} \propto \sqrt{\frac{L}{C}} \cdot \frac{L}{C}$ is large because locomotive is inductive load, so voltage is also very large after multiply by a small current.

$\mathrm{U}_{\mathrm{m}}$ can be expressed further as in

$$
U_{m}=\sqrt{I_{0}^{2} \frac{L}{C}+U_{0}^{2}}=\sqrt{I_{m}^{2} \sin ^{2} \theta \frac{L}{C}+U_{m}^{2} \cos ^{2} \theta}
$$

From (6), overvoltage is related to the ground inductance, capacitance and the angle of current when $\mathrm{QF}_{1}$ is turned off, and the voltage reaches its maximum value when $\mathrm{QF}_{1}$ is turned off at $\theta= \pm 90^{\circ}$.

\subsection{Mechanism Analysis of Overvoltage Caused by Switching on}

When the locomotive arrives at $\mathrm{CG}_{2}$, the neutral section becomes uncharged in the short time when $\mathrm{QF}_{1}$ is turned off and $\mathrm{QF}_{2}$ is not turned on. In this case, the low voltage side of the main transformer of EMU and asynchronous motor group in the traction invertor and the auxiliary circuit still form a closed loop [12]. Current flows through the closed loop because parts of the asynchronous motor are rotating and still remain energized. It becomes coupling in the high voltage side of main transformer through auxiliary winding, which represents residual voltage of the neutral section. Due to the transient difference in voltage between the residual voltage of neutral section and the voltage of power supply, impedance of transmission line and capacitance of neutral section appear oscillation process after $\mathrm{QF}_{2}$ is closed. Figure 4 is circuit schematic when $\mathrm{QF}_{2}$ is closed; Figure 5 is vector diagram of the closing circuit in which $\dot{u}_{c}, \dot{u}_{L}, \dot{u}_{R}$ and $\dot{u}_{B}$ represent the voltage of capacitance, inductance, resistance and power supply separately, $i$ represents current, $\beta$ represents phase angle of the power supply when switch is turned on, $\gamma$ represents impedance angle.

Since $\mathrm{L}_{\mathrm{N} 2}$ is very small when compared to the total inductance of the whole loop and the ground capacitance of neutral section $\mathrm{C}_{\mathrm{N}}$ is greater than which of locomotive $\mathrm{C}_{3}$, the function of $\mathrm{L}_{\mathrm{N} 2}, \mathrm{C}_{3}, \mathrm{C}_{2}, C_{2}^{\prime}$ can be ignored and circuit is equivalent to $\mathrm{RLC}$ circuit. Making $\mathrm{R}=\mathrm{R}_{\mathrm{S} 2}$ $+\mathrm{R}_{2}+\mathrm{R}_{\mathrm{N} 2}, \mathrm{~L}=\mathrm{L}_{\mathrm{S} 2}+\mathrm{L}_{2}, \mathrm{C}=\mathrm{C}_{\mathrm{N}}, \mathrm{u}_{\mathrm{B}}=\mathrm{U}_{\mathrm{m}} \sin (\omega \mathrm{t}+\beta)$, $\mathrm{KVL}$ equation of loop 1 can be got when $\mathrm{QF}_{2}$ is closed.

$$
L C \frac{d^{2} u_{c}}{d t^{2}}+R C \frac{d u_{c}}{d t}+u_{c}=U_{m} \sin (\omega t+\beta)
$$

This is a second order linear nonhomogeneous differential equation. The same method of solving zero-state response is used in full response of the second order circuit, which can be got by bringing a non-zero initial value into equation when confirming undetermined coefficient.

Characteristic equation can be expressed as follow.

$$
L C p^{2}+R C p+1=0
$$

characteristic root is

$$
P_{1,2}=-\frac{R}{2 L} \pm \sqrt{\left(\frac{R}{2 L}\right)^{2}-\frac{1}{L C}}=-a \pm \sqrt{a^{2}-\frac{1}{\omega_{0}^{2}}}
$$

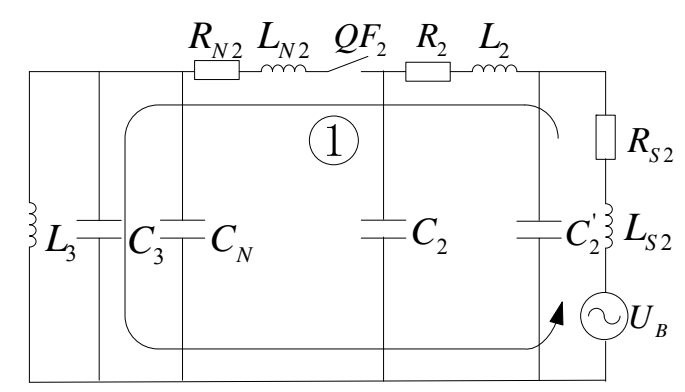

Figure 4. Schematic diagram of equivalent circuit when $\mathbf{Q F}_{2}$ is turned on.

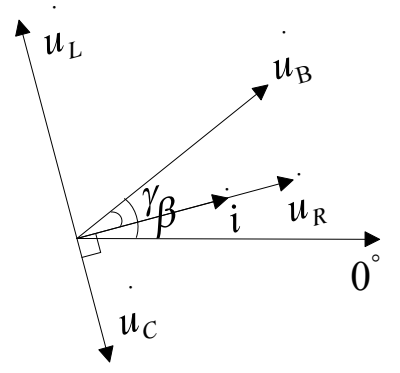

Figure 5. Vector diagram of equivalent circuit. 
where, $a=\frac{R}{2 L}$ represents attenuation coefficient of line, $\omega_{0}=\frac{1}{\sqrt{L C}}$ represents resonance angular frequency of line.

general solution as in

$$
u_{c h}=K_{1} e^{p_{1} t}+K_{2} e^{p_{2} t}
$$

particular solution as in

$$
u_{c p}=\frac{U_{m}}{Z} X_{c} \sin \left(\omega t+\beta-\gamma-\frac{\pi}{2}\right)
$$

where,

$$
Z=\sqrt{\left(X_{L}-X_{C}\right)^{2}+R^{2}}, \gamma=\arctan \frac{X_{L}-X_{C}}{R}
$$

Supposing $\mathrm{QF}_{1}$ is turned off and $\mathrm{QF}_{2}$ is turned on when $\mathrm{t}=0$, initial condition is

$$
\left\{\begin{array}{l}
u_{c}\left(0_{+}\right)=u_{c}\left(0_{-}\right)=u_{c 0} \\
\left.\frac{d u_{c}(t)}{d t}\right|_{t=0_{+}}=\frac{1}{C} i_{L}\left(0_{+}\right)=0
\end{array}\right.
$$

According to initial condition

$$
\begin{aligned}
u_{c h}= & \frac{u_{c 0}}{P_{2}-P_{1}}\left(P_{2} e^{p_{1} t}-P_{1} e^{p_{2} t}\right)+ \\
& \frac{U_{m}}{Z} X_{c}\left[\frac{\cos (\omega t+\beta-\gamma)}{P_{2}-P_{1}}\left(P_{2} e^{p_{1} t}-P_{1} e^{p_{2} t}\right)\right. \\
& \left.+\frac{\omega \sin (\omega t+\beta-\gamma)}{P_{2}-P_{1}}\left(e^{p_{1} t}-e^{p_{2} t}\right)\right]
\end{aligned}
$$

Because the resistance consumes electrical energy in the circuit, $u_{c}$ is a oscillation which attenuates periodically.

$$
a<\omega_{0} \text {, make } \frac{1}{L C}-\left(\frac{R}{2 L}\right)^{2}=\omega_{1}^{2}
$$

Equation (12) can also be expressed

$$
\begin{aligned}
u_{c h}= & \frac{\omega_{0} u_{c 0}}{\omega_{1}} e^{-a t} \sin \left(\omega_{1} t+\delta\right)+ \\
& \frac{U_{m}}{Z} X_{c} \frac{e^{-a t}}{\omega_{1}}\left[-\omega \sin \omega_{1} t \sin (\omega t+\beta-\gamma)+\right. \\
& \left.\omega_{0} \sin \left(\omega_{1} t+\delta\right) \cos (\omega t+\beta-\gamma)\right]
\end{aligned}
$$

where, $\delta=\arctan \frac{\omega_{1}}{a}$

Entire response is

$$
\begin{aligned}
u_{c}= & \frac{U_{m}}{Z} X_{c}\left\{\sin \left(\omega t+\beta-\gamma-\frac{\pi}{2}\right)+\frac{\omega_{0} u_{c 0}}{\omega_{1}} e^{-a t} \sin \left(\omega_{t} t+\delta\right)\right. \\
& +\frac{U_{m}}{Z} X_{c} \frac{e^{-a t}}{\omega_{1}}\left[-\omega \sin \omega_{q} t \sin (\omega t+\beta-\gamma)\right. \\
& \left.+\omega_{0} \sin \left(\omega_{1} t+\delta\right) \cos (\omega t+\beta-\gamma)\right]
\end{aligned}
$$

From (14), the voltage of neutral section relates to amplitude of power supply voltage, phase angle of the power supply when switch is turned on and residual voltage of neutral section in the moment $\mathrm{QF}_{2}$ is turned on. The voltage of neutral section is superimposition of steady-state component and transient component, so the overvoltage is higher. Transient component attenuates cyclically by coefficient $e^{-a t}$ due to the consumption of the line.

\subsection{Mechanism Analysis of Impact Current}

Voltage and prodigious impact current which is inseparable with the flux of locomotive main transformer are appearing in the process of changing-over between $\mathrm{QF}_{1}$ and $\mathrm{QF}_{2}$ when EMU is passing by phase-separation with electric load. Assuming that there is residual voltage in neutral section when $\mathrm{QF}_{1}$ is turned off, It is $u_{z}=$ $U_{z m} \sin \left(\omega_{z} t+\varphi\right) \quad\left(\omega_{Z}\right.$ represents angular frequency). As we all know, the relationship between voltage and flux is $u=N \frac{d \phi}{d t}$ (N represents the number of coils). Flux of locomotive main transformer can be obtained as follow.

$$
\phi_{z}=-\frac{U_{z m}}{N \omega_{z}} \cos \left(\omega_{z} t+\varphi\right)+C_{z}
$$

Where, $\mathrm{C}_{Z}$ represents attenuation non-periodic component.

When the $\mathrm{QF}_{2}$ is turned on, flux of locomotive as in

$$
\phi_{1}=-\frac{U_{m}}{N \omega} \cos (\omega t+\beta)+C_{1}
$$

Supposing that $\mathrm{QF}_{2}$ is turned on in $\mathrm{t}_{1}$. According to the principle of flux cannot be mutation

$$
\begin{gathered}
\phi_{1}\left(t=t_{1}\right)=\phi_{2}\left(t=t_{1}\right) \\
-\frac{U_{m}}{N \omega} \cos \left(\omega t_{1}+\beta\right)+C_{1}=-\frac{U_{m z}}{N \omega_{z}} \cos \left(\omega_{z} t_{1}+\varphi\right)+C_{z}
\end{gathered}
$$

Make (17) into (16)

$$
\begin{aligned}
\phi_{1}= & -\frac{U_{m}}{N \omega} \cos (\omega t+\beta)-\frac{U_{z m}}{N \omega_{z}} \cos \left(\omega_{z} t_{1}+\varphi\right) \\
& +\frac{U_{m}}{N \omega} \cos \left(\omega t_{1}+\beta\right)+C_{z}
\end{aligned}
$$

From (18), flux of locomotive main transformer is made up of steady-state flux and flux generated by DC component when $\mathrm{QF}_{2}$ is turned on. The residual voltage of neutral section decays with time in reality. So the flux is expressed as in

$$
\phi_{1}=\phi_{s}+\phi_{d}+\phi_{d t}
$$

where, $\Phi_{\mathrm{s}}$ represents steady-state flux, $\Phi_{\mathrm{d}}$ represents steady-state flux generated by DC component and $\Phi_{\mathrm{dt}}$ 
represents damped flux generated by DC component.

$$
I=\frac{\phi_{1} R_{m}}{N}
$$

where, $\mathrm{N}$ represents the number of coils and $R_{m}$ represents total reluctance.

When $\beta$ meet $\omega \mathrm{t}+\beta=\beta+2 \mathrm{kT}$, $\Phi_{1}$ will reach the maximum $2 \Phi_{\mathrm{s}}+\Phi_{\mathrm{dt}}$, and inrush current increases greatly. Impact current which up to 4-6 times as much as the rated current in closing side is the superposition of the inrush current and low-voltage side current.

\section{Simulation of EMU Passing by Phase-separation}

\subsection{Simulation Analysis of Overvoltage}

Making use of Matlab/Simulink to establish the module of passing by phase-separation with electric load. Simulation parameters are shown as follow. $\mathrm{R}_{\mathrm{S} 1}=0.6969 \Omega$, $\mathrm{L}_{\mathrm{S} 1}=4.17 \mathrm{mH}, \mathrm{R}_{\mathrm{S} 2}=0.3676 \Omega$ and $\mathrm{L}_{\mathrm{S} 2}=24.9 \mathrm{mH} ; \mathrm{R}_{1}=$ $0.48 \Omega, \mathrm{L}_{1}=4.76 \mathrm{mH}, \mathrm{R}_{2}=0.3836 \Omega$ and $\mathrm{L}_{2}=3.73 \mathrm{mH}$; $\mathrm{R}_{\mathrm{N}}=0.151 \Omega$ and $\mathrm{L}_{\mathrm{N}}=1.47 \mathrm{mH} ; \mathrm{C}_{\mathrm{N}}=0.01155 \mu \mathrm{F}, \mathrm{C}_{\mathrm{N} 1}=$ $0.000325 \mu \mathrm{F}$ and $\mathrm{C}_{\mathrm{N} 2}=0.00415 \mu \mathrm{F}$.

\subsubsection{Simulation Analysis of Overvoltage Caused by Switching off}

When $\mathrm{QF}_{1}$ is turned off, the simulation results of overvoltage are shown in Figure 6: Figure 6(a) shows the voltage waveform when current crossing zero $\left(\theta=0^{\circ}\right)$; Figure 6(b) shows the voltage waveform when the amplitude of current is maximum $\left(\theta= \pm 90^{\circ}\right)$. For inductive load, the phase angle of voltage is ahead of which of the current $90^{\circ}$, so the angle of $\mathrm{Ua}$ is $90^{\circ}$ when $\theta=0^{\circ}$.

The simulation results in Figure 6 indicate that $\mathrm{QF}_{1}$ turned off causes a large overvoltage whose maximum can reach $77.41 \mathrm{kV}$. The amplitude of the overvoltage is related to the phase of current when $\mathrm{QF}_{1}$ is turned off, which is a good description of the correctness of the analysis of the mechanism.

\subsubsection{Simulation Analysis of Overvoltage Caused by Switching on}

When $\mathrm{QF}_{2}$ is turned on in this process, the absolute value of the maximum voltage of neutral section which is shown in Table 1 can be got through simulating in the condition that phase angle $\beta$ is $0^{\circ}-350^{\circ}$.

As can be seen from I, overvoltage minimum appears when $\beta$ is in the vicinity of $10^{\circ}$ or $180^{\circ}$; overvoltage maximum is appearing when $\beta$ in the vicinity of $90^{\circ}$ or $270^{\circ}$.

The waveforms of overvoltage minimum and maximum are shown in Figure 7: Figure 7(a) $\beta=180^{\circ}$, Figure 7(b) $\beta=270^{\circ}$.
The simulation results in Figure 7 indicate that $\mathrm{QF}_{2}$ turned on causes a large overvoltage whose maximum can reach $77.68 \mathrm{kV}$. The amplitude of the overvoltage is related to the phase of power supply voltage and operating conditions when electromagnetic transient process occurs.

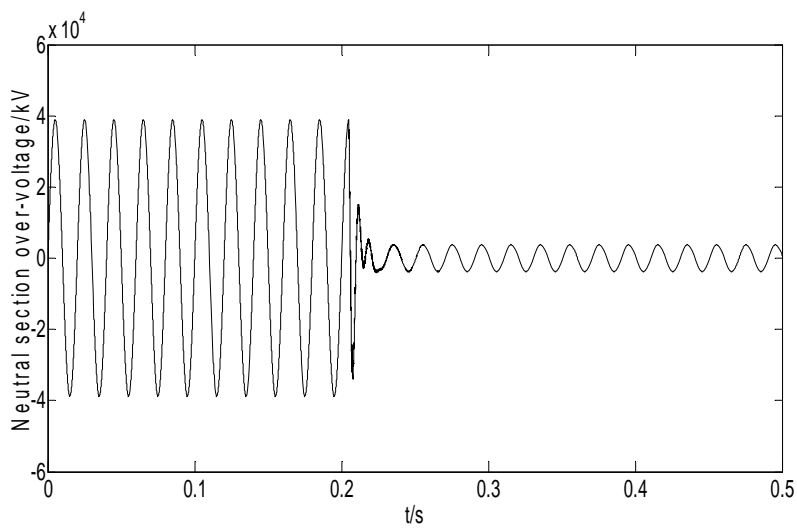

(a) $\mathrm{QF}_{1}$ is turned off when the phase angle of $\mathrm{Ua}$ is $90^{\circ}\left(\theta=0^{\circ}\right)$

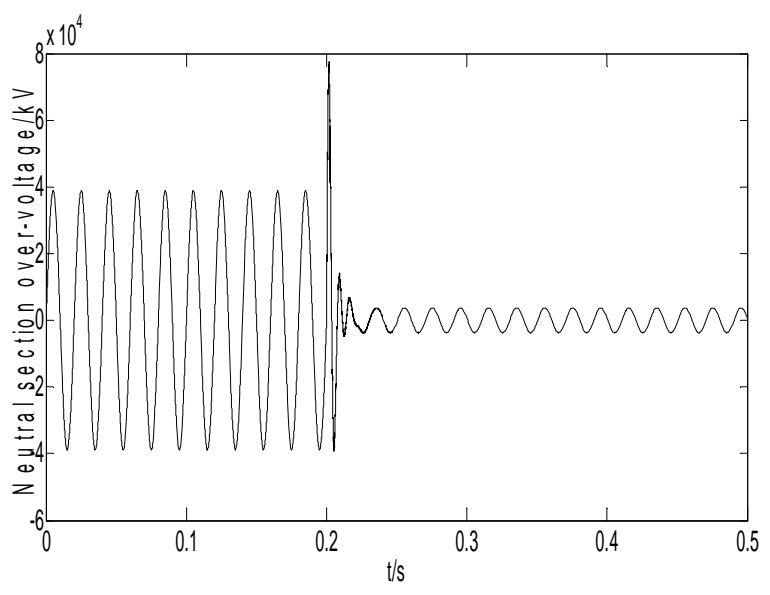

(b) $\mathrm{QF}_{1}$ is turned off when the phase angle of $\mathrm{Ua}$ is $0^{\circ}\left(\theta=-90^{\circ}\right)$

Figure 6. Overvoltage waveforms when $\mathrm{QF}_{1}$ is turned off.

Table 1. Absolute value of peak overvoltage of neutral section..

\begin{tabular}{ccccccc}
\hline$\beta\left(^{\circ}\right)$ & 0 & 10 & 20 & 30 & 40 & 50 \\
\hline $\mathrm{U}(\mathrm{kV})$ & 45.90 & 40.92 & 41.94 & 47.23 & 52.53 & 57.60 \\
$\beta\left(^{\circ}\right)$ & 60 & 70 & 80 & 90 & 100 & 110 \\
$\mathrm{U}(\mathrm{kV})$ & 62.23 & 66.25 & 69.48 & 71.11 & 70.48 & 67.60 \\
$\beta\left(^{\circ}\right)$ & 120 & 130 & 140 & 150 & 160 & 170 \\
$\mathrm{U}(\mathrm{kV})$ & 62.58 & 58.29 & 56.09 & 53.15 & 49.62 & 45.38 \\
$\beta\left(^{\circ}\right)$ & 180 & 190 & 200 & 210 & 220 & 230 \\
$\mathrm{U}(\mathrm{kV})$ & 40.60 & 42.15 & 47.50 & 52.92 & 58.38 & 63.64 \\
$\beta\left({ }^{\circ}\right)$ & 240 & 250 & 260 & 270 & 280 & 290 \\
$\mathrm{U}(\mathrm{kV})$ & 68.45 & 72.64 & 76.05 & 77.68 & 77.05 & 74.18 \\
$\beta\left(^{\circ}\right)$ & 300 & 310 & 320 & 330 & 340 & 350 \\
$\mathrm{U}(\mathrm{kV})$ & 69.15 & 62.93 & 60.85 & 58.02 & 54.64 & 50.56 \\
\hline
\end{tabular}




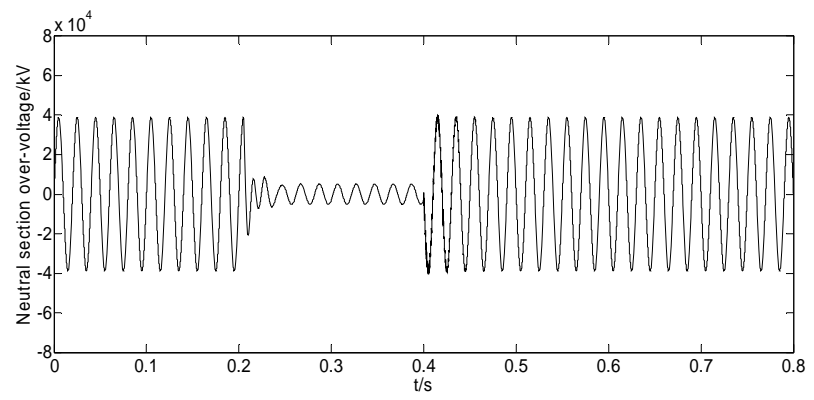

(a) $\mathrm{QF}_{2}$ is turned on when the phase angle of $\mathrm{Ub}$ is $180^{\circ}$

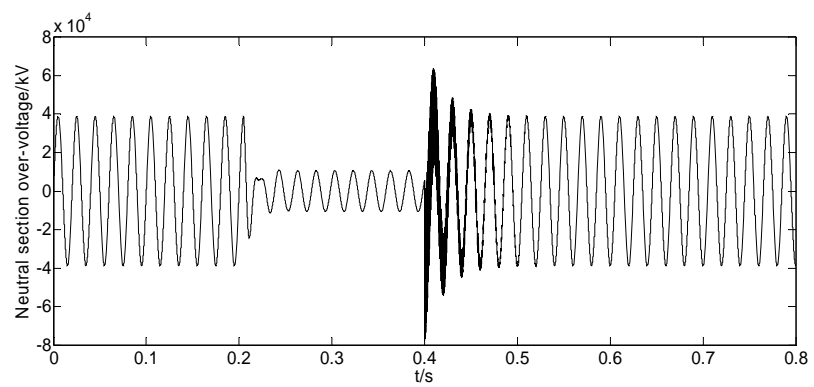

(b) $\mathrm{QF}_{2}$ is turned on when the phase angle of $\mathrm{Ua}$ is $270^{\circ}$

Figure 7. Overvoltage waveforms when $\mathbf{Q F}$ is turned on.

The protection gap on the locomotive roof discharges because of overvoltage, which results in traction substation tripping and interrupting power supply and transportation. The frequent shocks of overvoltage have an influence on the life of traction transformers, converter and auxiliary motor system. The overvoltage is related to oscillation caused by the changes in circuit parameters when circuit breaker is operating and voltage phase angle when switching on or off, thereby it can be suppressed effectively by eliminating oscillation in the condition of changing the circuit parameters. In addition, using phasecontrolled rectification technology in switching on and off is an effective method.

\subsection{Simulation Analysis of Impact Current Influence on Differential Protection}

Capacity of traction transformer adopted in simulation is 40 MVA, variable ratio is $220 / 27.5 \mathrm{kV}$; equivalent resistance and inductance of feeding section in both sides of phase-separation are $4.475 \Omega$ and $19.35 \mathrm{mH}$; equivalent resistance and inductance of neutral section is $0.063 \Omega$ and $0.198 \mathrm{mH}$; capacity of main transformer of EMU is 8MVA, variable ratio is $27.5 / 1.5 \mathrm{kV}$. Excitation inrush current of the EMU transformer is shown in Figure 8 when $\mathrm{QF}_{2}$ is turned on, and inrush current of the EMU transformer closing side is shown in Figure 9.

As can be seen from the simulation results: the core of EMU main transformer is saturation under the action of residual voltage of neutral section and overvoltage caused by switching off, which comes into being excitation inrush current whose amplitude is high and waveform exists obvious discontinuity angle when $\mathrm{QF}_{2}$ is turned on. Impact current whose amplitude is 5 times higher than that of rated current in closing side is the superposition of the inrush current and low-voltage side current. Waveform does not exist obvious discontinuity angle, however waveform generates obvious distortion because of nonperiodic component.

Making use of the CT model in PSCAD, impact current is flowing into the low-voltage side of traction transformer directly, and the secondary current of high and low sides of traction transformer can be got. CT parameter settings: $\mathrm{CT}$ ratio of low-voltage side $\mathrm{k}=2000 / 5$ $\mathrm{A}, 1=0.5 \mathrm{~m}, \mathrm{~S}=51.2 \mathrm{~cm}^{2}, \mathrm{R}=0.13 \Omega$; CT ratio of high-voltage side $\mathrm{k}=250 / 5 \mathrm{~A}, \mathrm{l}=0.7 \mathrm{~m}, \mathrm{~S}=23.2 \mathrm{~cm}^{2}, \mathrm{R}$ $=0.06 \Omega$. The impact current is imported into the primary side of CT, secondary current of CT is shown in Figure 10.

From Figure 10, due to the fact that the flux of CT core does not mutation, the waveform of secondary current does not show obvious distortion in the moment of closing. Then the waveform of secondary current is distorted because of CT core saturation, which is under the action of non-periodic component. The transmission characteristics of traction transformer between steadystate cycle component and non-periodic component is different, so there is a large difference in the non-periodic component of both sides. The non-periodic component of current in high-voltage side is much smaller than

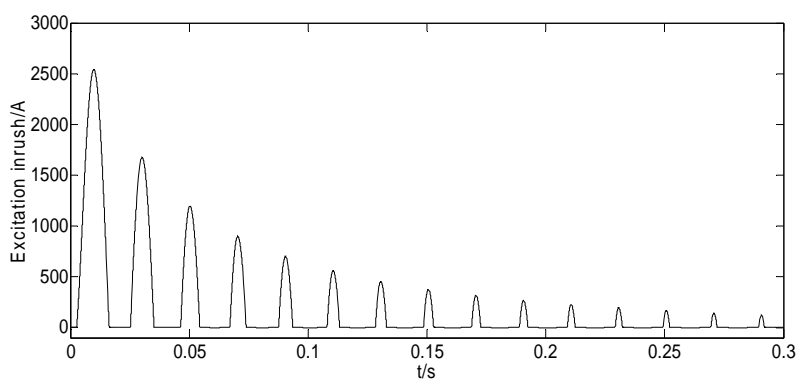

Figure 8. Excitation inrush current of the EMU transformer.

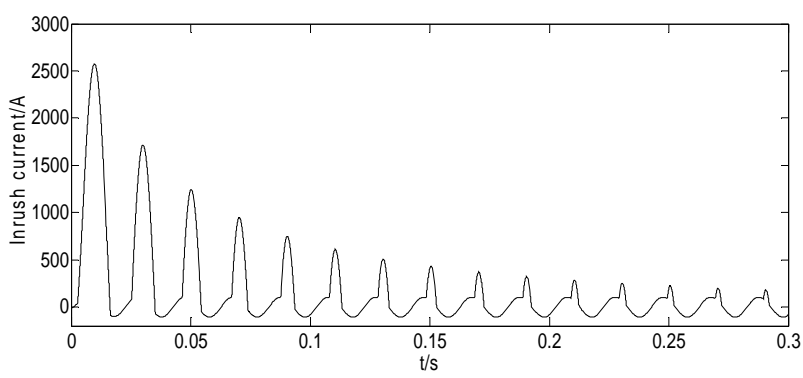

Figure 9. Inrush current of the EMU transformer closing side. 
that of current in low-voltage side. Therefore, the waveform of CT secondary current of high-voltage side does not contain any distortion.

Figure 11 shows waveform of the differential current flowing in the differential relay, which indicates that the differential current distortion within the first few cycles after closing is obvious, primarily because that the nonperiodic component of impact current is not attenuated sufficiently. After a period of time, the non-periodic component of the current is almost attenuation finished. The second harmonic of the differential current is analyzed by using Fourier transformation, and the result is shown in Figure 12.

Figure 12 shows that in the first cycle, the second harmonic content is reducing continuously, which is related to that CT secondary current of low-voltage side of traction transformer does not appear obvious distortion in

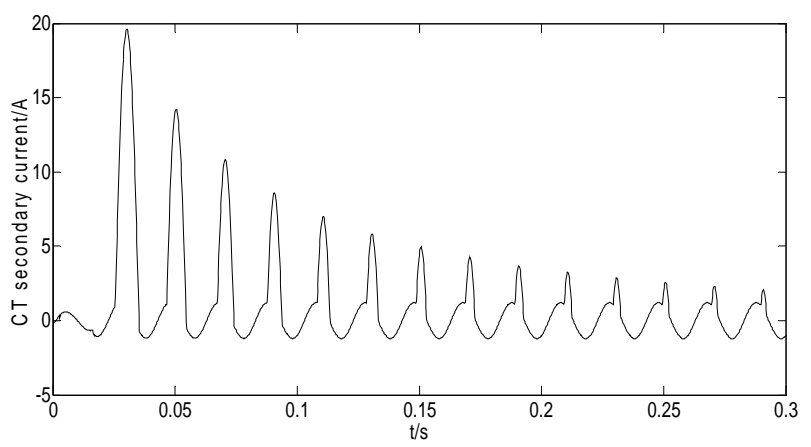

(a) CT secondary current in low voltage side of traction transformer

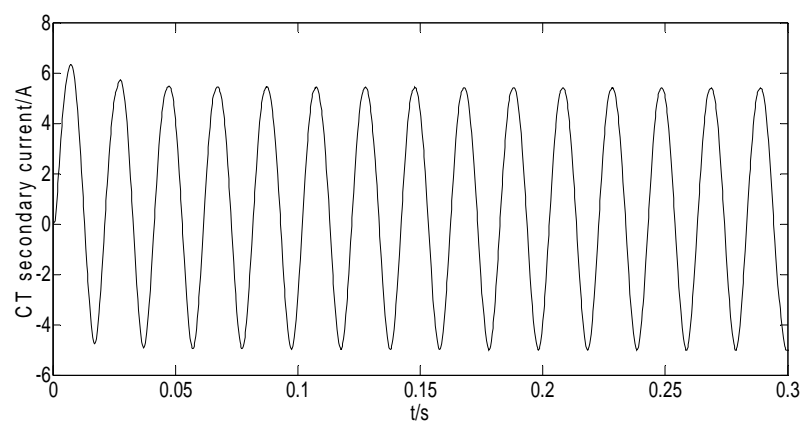

(b) CT secondary current in high voltage side of traction transformer

Figure 10. CT secondary current.

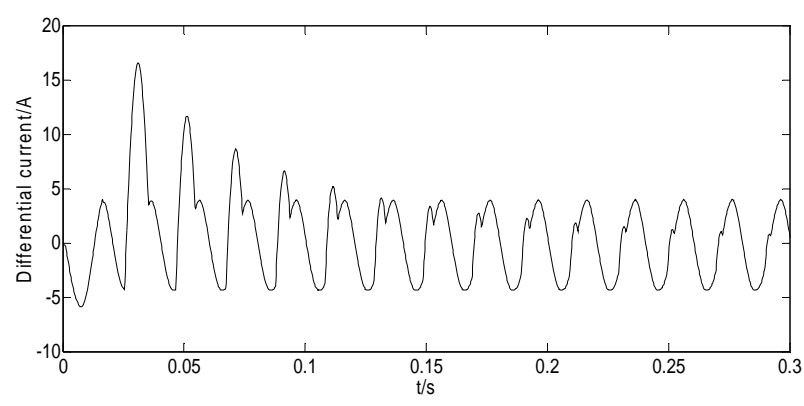

Figure 11. Waveform of the differential current.

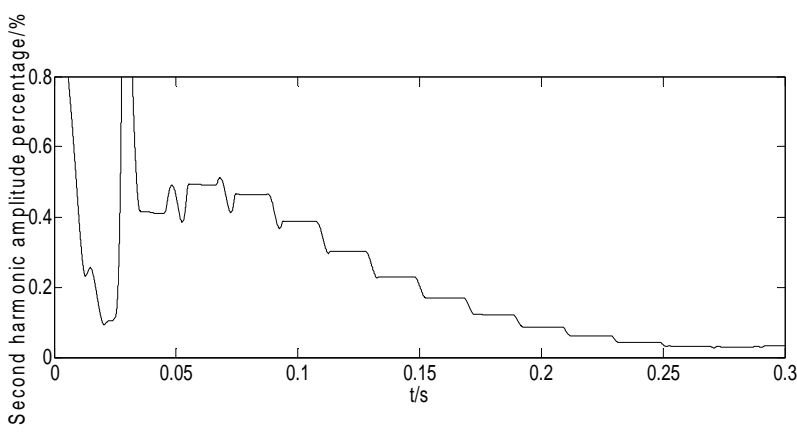

Figure 12. Second harmonic amplitude percentage in differential current.

the first cycle. Subsequently, the second harmonic content increases with the distortion of the waveform. The second harmonic content is less than $15 \%$ below its set value $(15 \%$ to $20 \%)$ in $0.15 \mathrm{~s}$ after closing, so protection cannot latch and differential protection of traction transformer is misoperation.

According to the analysis above, the impact current can cause traction transformer differential protection misoperation. For the misoperation caused by that second harmonic content is low, using dual scheme in differenttial protection can solve the problem by increasing the waveform recognition atresia criterion.

\section{Conclusions}

Mechanism of overvoltage and impact current in the electromagnetic transient process of passing by phase-separation with electric load is studied in the round in this article, and the equivalent model is built for each of the transient process, which can be used to analyze overvoltage and impact current. Suppressing overvoltage by changing the parameters of the circuit and using phase-controlled rectifier technology is proposed; differential protection misoperation caused by impact current is solved by increasing the waveform recognition atresia criterion. So the article provides a reference for subsequent studies, and the resonance overvoltage in this transient process is more complicated, which needs further analysis in later period.

\section{Acknowledgements}

The authors express sincere gratitude to the support of China National Natural Science Fund (50907055, U1134205 and 51177139) and Fundamental Research Funds for the Central Universities.

\section{REFERENCES}

[1] X. C. Chen, "Railway Power Supply Relay Protection and Automation," China Railway Press, 2010.

[2] J. Z. Wei, X. X. Guo and Z. Q. Han, "Protection on the 
Condition of EMU Passing by Phase-separation with Electric Load in High-speed Railway,"APAP2011, 2011,10.

[3] Z. Q. Han, "Study on Dynamic Process and Feeder Protection on the Condition of EMU Passing by Phase- separation with Electric Load in High-speed Railway," Academic trends, Vol. 4, 2008, pp.10-12.

[4] Z. Z. Li, "Analysis of Causes of Tripping during Electric locomotive's Passage of Neutral Section and Study of Its Prevention Measures," Electric Railway, Vol. 4, 2008, pp. 10-12.

[5] S. B. Gao, Y. F. Wang and J. Zhang, "Analysis on Fault and Operating Features of Feeder Protection Against Wrong-phase Short Circuit for AC Traction Substation," Journal of The China Railway Society, Vol. 4, 2000, pp. 24-27.

[6] Y. H. Guo, J. S. Lian and K. L. Zhang, "Influnce of Auto-passing of Neutral Section on Electric Locomotive," Electric Drive For Locomotive, Vol. 2, 2000, pp. 13-15.

[7] G. Ma, G. N. Wu and T. Wang, "Simulation of Excitation Surge Current in Electric Locomotive and Analysis of In- fluence to Public Grid," Electric Railway, Vol. 1, 2010 , pp.1-4.

[8] W. Ran, X. Li and B. Liu, "Research on Transient Process of Ground's Auto-Passing Neutral Section at Switching Time," Transactions of China Electrotechnical Society, Vol.11, 2011, pp. 150-154.

[9] L. Q. Luo, Y. Wang and G. Chang, "Analysis of Inrush Current during Auto-passing Phase of Electric Locomotive," Journal of Beijing Jiaotong University, Vol. 6, 2011, pp.57-61.

[10] H. L. Weng, X. N. Lin and P. Liu, “A Novel Explanation to the Mal-operation of Differential Protection during the Loaded Transformer Energization," Proceeding of the CSEE, Vol. 26, 2006, pp. 27-32.

[11] W. Q. Sun, S. X. Shan and G. F. Zheng, "Comparsion between Home and Aboard of Automatic Passing over of Neutral Section Device," Electric Railway, Vol. 2, 2002, pp. 12-16.

[12] S. B. Yang, M. Huang and M. L. Wu, "High Speed EMU Modeling Based on the Measured Data," Sustainable Power Generation and Supply, 2009. 